\section{Acne exacerbation after tofacitinib treatment for alopecia areata}

\author{
Sawsan Alharthi, ${ }^{1}$ Mohammed G. \\ Turkmani, ${ }^{2}$ Mohammed I. AlJasser ${ }^{1}$ \\ ${ }^{1}$ Division of Dermatology, King Saud \\ Bin Abdulaziz University for Health \\ Sciences, Riyadh; ${ }^{2}$ Derma Clinic, \\ Riyadh, Saudi Arabia
}

\begin{abstract}
The major discovery of the novel therapeutic Janus kinase (JAK) inhibitors has been implicated in several dermatological diseases. Recently, studies have shown the efficacy and encouraging results of oral JAK inhibitors as a treatment for alopecia areata (AA). Due to the novelty of this treatment, potential side effects are not fully explored. In this paper, we present a case of a 28 -year-old male with a 10 -year history of alopecia totalis (AT) treated successfully with tofacitinib with encouraging effects on hair regrowth; however, a significant worsening of the patient's facial acne was observed four months after AT treatment initiation. JAK inhibitors have promising results in the management of different dermatological conditions including moderatesevere forms of AA with few reported adverse events. Acne exacerbation is a unique observed adverse effect of this therapy. More thorough larger sized studies are needed to further characterize the association between acne exacerbation and the use of JAK inhibitors.
\end{abstract}

\section{Introduction}

The advances in the understanding of the pathogenesis of several dermatologic diseases have empowered the development of new targeted therapeutic approaches. ${ }^{1}$ In the past few years, the safety and efficacy of oral Janus Kinase (JAK) inhibitors in dermatology has been a topic of a great interest. Previous studies reported promising outcomes of JAK inhibitors in the treatment of psoriasis, atopic dermatitis, and vitiligo. Recent insights have shown encouraging results of JAK inhibitors in inducing hair regrowth in patients with moderate-severe variants of alopecia areata (AA) such as alopecia totalis (AT) and alopecia universalis $(\mathrm{AU}){ }^{2}$
JAKs are considered an intracellular enzyme that bind to one or more of the cytoplasmic domains of cytokine receptors and activate the JAK/STAT pathway. The JAK expression and signaling pathway is known to be involved in many inflammatory skin diseases. Four JAK isoforms are currently known: JAK1, JAK2, JAK3, and TYK2. ${ }^{3}$ JAK inhibitors are drugs that inhibit the activity of one or more of the JAK enzymes; therefore, it interferes with the pathway of intracellular signaling. First-generation JAK inhibitors such as tofacitinib, ruxolitinib, and baricitinib block multiple JAK enzymes, while the second generation like decernotinib target a specific JAK. ${ }^{4}$ Many indications exist for the use of JAK inhibitors; however, psoriatic arthritis represents the only skin $\square$ related disease for which oral tofacitinib is currently approved. ${ }^{5}$

Due to the novelty of this therapy, potential side effects are not fully explored. Systemic administration of JAK inhibitors can be associated with several adverse reactions with infections being the most worrisome. Other rare but possible side effects are anemia, thrombocytopenia, neutropenia, and deranged lipid profile. ${ }^{6,7}$

Although the major discovery of the novel therapeutic JAK inhibitors has been implicated in several dermatological diseases, limited studies reported the dermatological side effects of JAK inhibitors. This paper narrates a case of a possible effect of tofacitinib on acne exacerbation in a patient treated successfully for alopecia totalis (AT) using tofacitinib therapy.

\section{Case Report}

An otherwise healthy 28-year-old male presented with a 10 -year history of alopecia totalis characterized by complete loss of scalp hair. He was previously treated with minoxidil 5\% solution, topical corticosteroids, and topical anthralin with mild improvement. Tofacitinib $5 \mathrm{mg}$ twice daily was therefore initiated due to the severity of his condition and lack of response to other therapies. Four months later, he showed excellent hair regrowth. However, his baseline mild acne significantly worsened (Figure 1). Acne was treated with doxycycline $100 \mathrm{mg}$ twice daily for one month followed by $100 \mathrm{mg}$ once daily for another month. This resulted in a significant improvement in acne. Adapalene $0.1 \%$ cream was used a maintenance therapy with no recurrence of acne. Tofacitinib was continued at the same dose.
Correspondence: Sawsan Alharthi, College of Medicine, King Saud Bin Abdulaziz University for Health Sciences, Riyadh, Saudi Arabia.

Tel: +966-506004258

E-mail: dr.sawsanalharthi@gmail.com

Key words: Tofacitinib; JAK; Acne.

Contributions: The authors contributed equally.

Conflict of interest: The authors declare no potential conflict of interest.

Funding: None.

Ethical approval and consent to participate: Written informed consent was obtained from the individual(s) for the publication of any potentially identifiable images or data included in this article.

Availability of data and material: Data and materials are available by the authors.

Please cite this article as: Alharthi S, Turkmani MG, Al Jasser MI. Acne exacerbation after tofacitinib treatment for alopecia areata. Dermatol Rep 2022;14:9396.

Received for publication: 7 October 2021. Accepted for publication: 12 October 2021.

This work is licensed under a Creative Commons Attribution-NonCommercial 4.0 International License (CC BY-NC 4.0).

(C) Copyright: the Author(s), 2022

Licensee PAGEPress, Italy

Dermatology Reports 2022; 14:9396

doi:10.4081/dr.2022.9396

Publisher's note: All claims expressed in this article are solely those of the authors and do not necessarily represent those of their affiliated organizations, or those of the publisher, the editors and the reviewers. Any product that may be evaluated in this article or claim that may be made by its manufacturer is not guaranteed or endorsed by the publisher.

\section{Discussion}

Tofacitinib is one of the JAK $1 / 3$ inhibitors, which is an approved drug for treatment of several rheumatological diseases including rheumatoid and psoriatic arthritis. ${ }^{8}$ In the last few years, several studies investigated the efficacy of JAK inhibitors in the treatment of AA. ${ }^{9}$ A study of 90 patients with AT and AU treated with tofacitinib showed encouraging results and few reported adverse events. ${ }^{10}$ In an openlabel study of 75 patients treated with either tofacitinib or ruxolitinib, hair regrowth was 

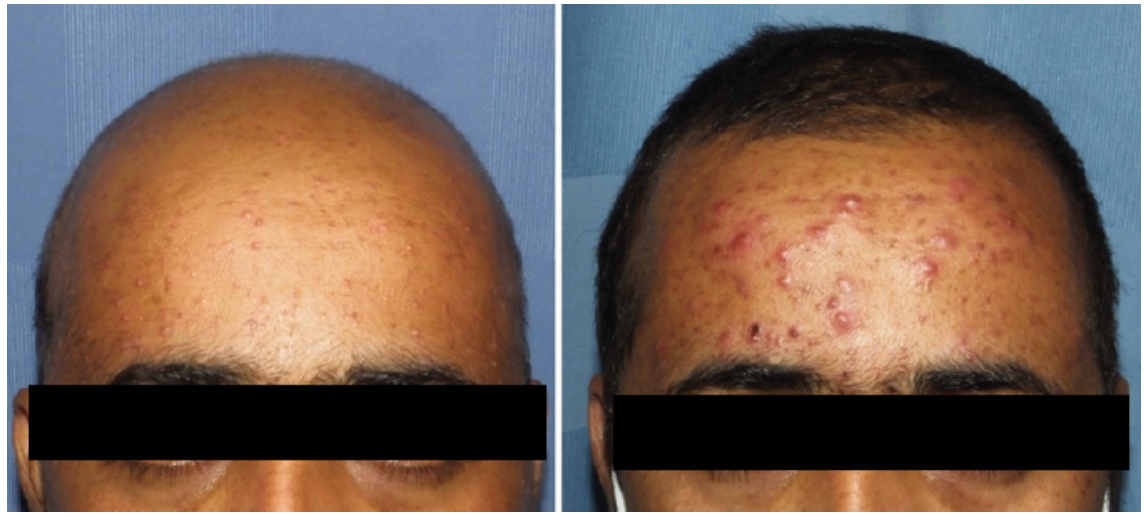

Figure 1. Patient with alopecia totalis and mild acne before the initiation of tofacitinib (Left). Excellent hair regrowth and significant worsening of acne 4 months after starting tofacitinib (Right).

achieved with both medications. ${ }^{11}$ Similarly, the outcome in our patient was very successful and encouraging and the result on hair regrowth was visible and profound.

Interestingly, our patient had a mild form of acne prior the use of tofacitinib. A significant worsening of his acne was noticed four months after the initiation of tofacitinib therapy. The association of acne with the use of tofacitinib and other JAK inhibitors therapy has been reported in few studies. A recent meta-analysis of 275 patients assessed the benefit and risk profile of tofacitinib in AA patients. Acne was one of the most reported adverse effects $(13.2 \%){ }^{12}$ Likewise, acne was reported in $7.8 \%$ of patients treated with tofacitinib for AA. ${ }^{10}$ In contrast, another meta-analytical study involving 3402 of atopic dermatitis patients treated with different drugs of JAKs inhibitors, none of the patients developed acne with the use of tofacitinib. Nonetheless, some patients experienced acne occurrence with other selective JAK1 and/or JAK2 inhibitors, mainly with upadacitinib, abrocitinib, and baricitinib. ${ }^{13}$ Rothstein B, evaluated the use of topical ruxolitinib, another JAK inhibitor, in patients with vitiligo. Two out of 11 patients developed a transient papular eruptions or worsening of acne. ${ }^{14}$ These studies suggest the possible positive association between acne development or worsening and the use JAK inhibitors. Although this potential side effect can be treated with standard acne therapy, it is essential to inform patients about the possibility of this situation to reduce the frustrations that might occur later.

The mechanism of acne exacerbation in patients on JAK inhibitors is still unclear. A case control study of 28 patients with acne vulgaris were compared to a control group. Biopsies of acne lesions showed an overex- pression of JAK1 and JAK3 compared to non-lesional skin in the control group. This proposes the involvement of JAK activation and signaling pathway in acne lesions. ${ }^{15}$ Because of these results, the study indicated the possibility of JAK involvement being a new target for therapy in acne patients. ${ }^{15}$ However, this contradicts the current observations of acne exacerbation and worsening among patients treated with JAK inhibitors. Unfortunately, little is known about JAK expression in acne and its relationship with JAK inhibitors. The literature is limited and does not answer many key questions. Larger studies are needed to further elucidate the role of JAK signaling and expression in acne.

\section{Conclusions}

The discovery of JAK inhibitors represents a major breakthrough in the management of different dermatological conditions including moderate-severe forms of AA with promising effects and few reported adverse events. Acne exacerbation is an additional observed effect of this therapy. More thorough larger sized studies are needed to further assess the possible association of increasing acne in patients treated with JAK inhibitors.

\section{References}

1. Solimani F, Meier K, Ghoreschi K. Emerging Topical and Systemic JAK Inhibitors in Dermatology. Front Immunol 2019;10:2847.

2. Damsky W, King BA. JAK inhibitors in dermatology: The promise of a new drug class. J Am Acad Dermatol 2017;76:736-44.
3. Samadi A, Ahmad Nasrollahi S, Hashemi A, et al. Janus kinase (JAK) inhibitors for the treatment of skin and hair disorders: a review of literature. J Dermatol Treat 2017;28:476-83.

4. Cinats A, Heck E, Robertson L. Janus Kinase Inhibitors: A Review of Their Emerging Applications in Dermatology. Skin Therapy Lett 2018;23:5-9.

5. Mahajan S, Hogan JK, Shlyakhter D, et al. VX-509 (decernotinib) is a potent and selective Janus kinase 3 inhibitor that attenuates inflammation in animal models of autoimmune disease. J Pharmacol Exp Ther 201;353:405-14.

6. Shreberk-Hassidim R, Ramot Y, Zlotogorski A. Janus kinase inhibitors in dermatology: A systematic review. J Am Acad Dermatol 2017;76:745-53.

7. Ramot Y, Zlotogorski A. [JAK inhibitors for the treatment of alopecia areata]. Harefuah 2020;159:38-42.

8. Kontzias A, Laurence A, Gadina M, O'Shea JJ. Kinase inhibitors in the treatment of immune-mediated disease. F1000 Med Rep 2012;4:5.

9. Jabbari A, Dai Z, Xing L, et al. Reversal of alopecia areata following treatment with the JAK1/2 inhibitor baricitinib. EBioMedicine 2015;2:351-5.

10. Liu LY, Craiglow BG, Dai F, King BA. Tofacitinib for the treatment of severe alopecia areata and variants: a study of 90 patients. J Am Acad Dermatol 2017;76:22-8.

11. Almutairi N, Nour TM, Hussain NH. Janus Kinase Inhibitors for the Treatment of Severe Alopecia Areata: An Open-Label Comparative Study. Dermatology 2019;235:130-6.

12. Guo L, Feng S, Sun B, et al. Benefit and risk profile of tofacitinib for the treatment of alopecia areata: a systemic review and meta-analysis. J Eur Acad Dermatol Venereol 2020;34:192-201.

13. Tsai HR, Lu JW, Chen LY, Chen TL. Application of Janus Kinase Inhibitors in Atopic Dermatitis: An Updated Systematic Review and Meta-Analysis of Clinical Trials. J Pers Med 2021;11: 279.

14. Rothstein B, Joshipura D, Saraiya A, et al. Treatment of vitiligo with the topical Janus kinase inhibitor ruxolitinib. J Am Acad Dermatol 2017;76:1054-60.

15. Awad SM, Tawfik YM, El-Mokhtar MA, et al. Activation of Janus kinase signaling pathway in acne lesions. Dermatol Ther 2021;34:e14563. 